\title{
Is ventilated hospital-acquired pneumonia a worse entity than ventilator-associated pneumonia?
}

\author{
Maria Sole Vallecoccia (10 1,2,5 , Cristina Dominedò (10 ${ }^{1,2,5}$, Salvatore Lucio Cutuli ${ }^{1,2}$ \\ Ignacio Martin-Loeches ${ }^{3,4}$, Antoni Torres $\mathbb{B}^{4}$ and Gennaro De Pascale ${ }^{1,2}$
}

\begin{abstract}
Affiliations: ${ }^{1}$ Dept of Anesthesiology, Intensive Care and Emergency Medicine, Fondazione Policlinico Universitario A. Gemelli IRCCS, Rome, Italy. ${ }^{2}$ Università Cattolica del Sacro Cuore, Rome, Italy. ${ }^{3}$ Dept of Intensive Care Medicine, Multidisciplinary Intensive Care Research Organization (MICRO), St James's Hospital, Dublin, Ireland. ${ }^{4}$ Service of Pneumology, Hospital Clinic of Barcelona, University of Barcelona, Institut d'Investigació August Pi i Sunyer (IDIBAPS) and Centro de Investigación Biomédica en Red, Enfermedades Respiratorias (CIBERES), Barcelona, Spain. ${ }^{5}$ Joint first authors.
\end{abstract}

Correspondence: Gennaro De Pascale, Fondazione Policlinico A. Gemelli IRCCS - Università Cattolica del Sacro Cuore Largo A. Gemelli 8, 00168 Rome, Italy. E-mail: gennaro.depascalemddamail.com

@ERSpublications

Due to the different mortality of each subtype of nosocomial pneumonia, including ventilatorassociated pneumonia and hospital-acquired pneumonia requiring mechanical ventilation, new prospective studies are urgently needed https://bit.ly/3fFoZ6U

Cite this article as: Vallecoccia MS, Dominedò C, Cutuli SL, et al. Is ventilated hospital-acquired pneumonia a worse entity than ventilator-associated pneumonia? Eur Respir Rev 2020; 29: 200023 [https:// doi.org/10.1183/16000617.0023-2020].

\section{ABSTRACT}

Introduction: Nosocomial pneumonia develops after $\geqslant 48 \mathrm{~h}$ of hospitalisation and is classified as ventilator-associated pneumonia (VAP) and hospital-acquired pneumonia (HAP); the latter may require mechanical ventilation (V-HAP) or not (NV-HAP).

Main findings: VAP and HAP affect a significant proportion of hospitalised patients and are characterised by poor clinical outcomes. Among them, V-HAP has the greatest 28-day mortality rate followed by VAP and NV-HAP (27.8\% versus $18 \%$ versus $14.5 \%$, respectively). However, no differences in terms of pathophysiology, underlying microbiological pathways and subsequent therapy have been identified. International guidelines suggest specific flow charts to help clinicians in the therapeutic management of such diseases; however, there are no specific recommendations beyond VAP and HAP classification. HAP subtypes are scarcely considered as different entities and the lack of data from the clinical scenario limits any final conclusion. Hopefully, recent understanding of the pathophysiology of such diseases, as well as the discovery of new therapies, will improve the outcome associated with such pulmonary infections.

Conclusion: Nosocomial pneumonia is a multifaced disease with features of pivotal interest in critical care medicine. Due to the worrisome data on mortality of patients with nosocomial pneumonia, further prospective studies focused on this topic are urgently needed.

Provenance: Submitted article, peer reviewed.

Received: 28 Jan 2020 | Accepted after revision: 14 June 2020

Copyright $\odot$ ERS 2020. This article is open access and distributed under the terms of the Creative Commons Attribution Non-Commercial Licence 4.0 . 
Introduction: what is new in the classification of nosocomial pneumonia

Nosocomial pneumonia is one of the most common causes of infection among patients admitted to the intensive care unit (ICU) and is associated with life-threatening complications and death in hospitalised patients [1]. Nosocomial pneumonia is defined as an infection of the lung which develops in patients admitted to the hospital for $\geqslant 48 \mathrm{~h}$ and includes ventilator-associated pneumonia (VAP) and hospitalacquired pneumonia (HAP). VAP is diagnosed in patients who have been mechanically ventilated for $\geqslant 48 \mathrm{~h}$, while HAP is independent from mechanical ventilation [2], although such intervention may be required to treat respiratory failure. Accordingly, HAP requiring mechanical ventilation (V-HAP) may be considered a specific subtype [3], which is characterised by an increased clinical severity (due to respiratory failure) compared to nonventilated HAP (NV-HAP).

Over the past two decades, many researchers have investigated VAP [4], leading to the implementation of preventive care bundles which have produced significant declines in its incidence, mortality and healthcare costs $[5,6]$. In contrast, although an increasing number of articles have been published on HAP [7], research into V-HAP is scarcely available.

In this review, we report the current evidence on the epidemiology of nosocomial pneumonia (e.g. main risk factors and microbiological aetiology), as well as its diagnosis and treatment, which come from meta-analyses, randomised controlled trials and any recent interventional or observational paper that we considered relevant for the topic. Specifically, we focus on articles which compared VAP and V-HAP to investigate the difference among them in terms of epidemiology, pathophysiology, diagnosis and outcome.

\section{Main findings \\ Epidemiology}

The incidence of VAP varies according to the characteristics of the study population [8], diagnostic criteria [9] and geographical origin. DUDECK et al. [10] reported an incidence of VAP rate of 1-2.5 cases per 1000 ventilator-days in North America. Conversely, Koulentr et al. [11] observed an incidence of 18.3 VAP episodes per 1000 ventilator-days in Europe. A recent article reported the incidence of HAP as 3.63 cases per 1000 patient-days in the United States [12]. In a prospective study on ICU-acquired pneumonia [13], $52 \%$ of cases were diagnosed as VAP and $48 \%$ as HAP. Specifically, $>50 \%$ of HAP cases required mechanical ventilation. Mortality and outcomes vary significantly between the different subtypes of nosocomial pneumonia [14]. In 2016, MiсEк et al. [15] observed that patients with HAP presented an 8.4-fold increased risk of death, an eight-fold increase in mechanical ventilation and a longer hospital length of stay compared with those not affected by HAP. More recently, seven clinical trial datasets on both VAP and HAP in patients admitted to ICU have been analysed [16]. V-HAP had the highest all-cause mortality rate at day $28(27.8 \%)$, followed by VAP (18\%) and NV-HAP (14.5\%).

Although the reasons for this difference in mortality are still unknown, and further studies are warranted to shed light on this matter, we believe that several factors may be involved. First, the spread of noninvasive ventilation (NIV) and high-flow oxygen therapy (HFOT) reduces the percentage of patients requiring immediate intubation, but increases the number of sick patients with risk factors for acquiring severe HAP [17] who could require subsequent intubation and mechanical ventilation. Moreover, compared with VAP, HAP poses additional challenges for respiratory sampling and, thus, for obtaining microbiological diagnosis [18], possibly causing a delay in the appropriate treatment, especially on the ward. Indeed, it has been demonstrated that rapid diagnosis and initiation of adequate empiric antimicrobial treatment are of paramount importance in the outcome of patients with nosocomial pneumonia [19-21]. Lastly, many care packages to prevent VAP have been implemented recently, leading to a reduction in incidence of VAP $[1,2,17]$. Their efficacy in improving the survival of patients with VAP has been demonstrated [22]. Conversely, little work has been done on HAP prevention, and the efficacy of most potential strategies remain unproven [23].

\section{Pathophysiology}

The pathophysiology of nosocomial pneumonia relies on the paradigm of pharyngeal bacterial aspirations, which considers the healthy distal airways to be sterile site that is contaminated by micro-aspiration of digestive contents during the hospital stay. Furthermore, patients admitted to hospital demonstrate a severe impairment of respiratory physiology [24], which is influenced by the patient's medical conditions (e.g. main disease and comorbidities) and therapeutic interventions (e.g. drugs or mechanical ventilation) [25]. The microbiological evidence of nosocomial pneumonia was more frequently available in patients with VAP compared with HAP [18]. However, in patients for whom the microbiological aetiology was known, pathogens were similar between VAP and HAP, suggesting that both types of patients might benefit from similar antimicrobial treatment. 


\section{Risk factors for multidrug-resistant pathogens}

The dramatic increase of multidrug-resistant (MDR), extensively drug-resistant and pandrug-resistant bacteria [26] has complicated the management and outcome of nosocomial pneumonia [27]. Empiric treatment of VAP and HAP should be guided by local epidemiology and susceptibility data, together with a careful assessment of individual risk factors for MDR pathogens. The 2016 Infectious Diseases Society of America (IDSA)/American Thoracic Society (ATS) guidelines [1] and the 2017 European Respiratory Society (ERS)/European Society of Intensive Care Medicine (ESICM)/European Society of Clinical Microbiology and Infectious Diseases (ESCMID)/Latin American Thoracic Association (ALAT) guidelines [2] provided a different algorithm for the empiric therapy of nosocomial pneumonia, based on patient- and centre-oriented risk factors for MDR pathogen infection [28]. According to the 2016 IDSA/ATS guidelines [1], risk factors for MDR infection in patients with VAP included previous intravenous antibiotic therapy within 90 days, hospitalisation for $\geqslant 5$ days before the diagnosis of VAP and organ failure (septic shock at the time of VAP diagnosis, acute respiratory distress syndrome and need for renal replacement therapy before VAP onset). Risk factors for MDR infection in patients with HAP have been investigated less, and only previous use of i.v. antibiotics has been identified in patients with HAP, without any distinction between NV-HAP and V-HAP [29]. This risk factor has been identified as the most predictive for VAP/HAP, specifically caused by methicillin-resistant Staphylococcus aureus (MRSA) [30, 31] and Pseudomonas aeruginosa [32].

In a validation study of the 2016 IDSA/ATS guidelines including 316 patients with VAP and HAP, EKREN et al. [33] observed a higher sensitivity compared with specificity for the majority of the risk factors reported. Previous i.v. antibiotic use and $\geqslant 5$ days of hospitalisation showed the highest negative predictive values for VAP (79\% and $80 \%$, respectively) and i.v. antibiotic use for HAP (69\%). Based on the role of local ecology [34] and the growing evidence that colonisation with MDR pathogens influences the occurrence of MDR pneumonia [35, 36], the 2017 ERS/ESICM/ESCMID/ALAT guidelines suggested taking into account hospital settings with high rates of MDR pathogens (>25\%) and previous colonisation with MDR pathogens to identify patients at increased risk of MDR infection [37]. However, the authors did not include acute respiratory distress syndrome and renal replacement therapy in the definition, since they have been related to severity of the disease rather than the risk for MDR pathogen infection. Nevertheless, we consider the risk of mortality due to the degree of organ dysfunction in order to implement empiric antibiotic therapy [38].

\section{Diagnosis}

The diagnosis of nosocomial pneumonia is based on the presence of a new pulmonary infiltrate on chest radiography or computed tomography together with some clinical features (such as fever or hypothermia, purulent secretions, oxygenation impairment) and laboratory data (leucocytosis or leukopenia). Theoretically, antibiotic choice should be based on the results of cultures of respiratory secretions [1]. Of course, if empiric antimicrobial therapy is necessary, the collection of a lower respiratory tract sample should be undertaken prior to the beginning of empiric treatment.

As discussed earlier, a higher rate of mortality in patients with bacterial V-HAP than those with bacterial VAP has been reported recently [16]. Indeed, HAP still represents a challenge for clinicians due to the difficulty of collecting a lower respiratory tract sample [7]. This can be done using an invasive technique such as fibreoptic bronchoscopy to collect a bronchoalveolar lavage. However, it requires the expertise of qualified clinicians and is not risk-free (e.g. pneumothorax, impact on gas exchange, haemodynamic instability). Due to these potential deleterious complications, the benefits of this technique are not clear [1]. Moreover, while it is easy and feasible to access the lower airways through an endotracheal tube, performing a bronchoscopy in a spontaneously breathing patient can be challenging, although several reports have proved the safety of this technique in patients on NIV or HFOT [39-42].

Alternatively, the culture of sputum is a noninvasive technique that can be obtained spontaneously, by induction via nasotracheal suctioning or endotracheal aspiration in intubated patients. Data on this practice are limited [7], due to the difficulty of obtaining an adequate sample and the need for patient collaboration and an assessment of quality and representativeness of the lower respiratory tract. Moreover, a positive result may only represent colonisation [7].

No recommendation was made on invasive or noninvasive diagnostic methods in VAP in the 2016 IDSA/ ATS guidelines [1]; since their impact on the outcome is comparable, there is no evidence regarding sampling in HAP, even though the same guidelines underline the importance of noninvasive respiratory sampling in nosocomial pneumonia rather than empiric treatment alone. This recommendation is graded as weak with low-quality evidence, based on a lack of research showing an improvement in patient-centred outcomes. Nevertheless, culture data may represent an opportunity for increasing appropriate coverage, thus minimising unnecessary antibiotic exposure. Accordingly, the collection of sputum must be encouraged in patients with nonventilated HAP, ensuring the quality and lower respiratory tract 
representativeness. This recommendation mainly comes from evidence on community-acquired pneumonia (CAP) [43], although some reports from HAP are promising [44].

In a recent observational study including 200 patients with HAP, 122 of whom required mechanical ventilation, patients assessed with an invasive diagnostic method had higher proportions of microbiological diagnosis [18]. Patients with microbiological diagnosis more frequently presented changes in their empiric antibiotic scheme, mainly de-escalation. Interestingly, the authors observed that severity, hypoxaemia and chest radiography patterns were associated with intubation after performing a fibreoptic bronchoscopy; in addition, they suggested the need to develop a prediction tool to evaluate which patients with HAP would need mechanical ventilation.

It has been demonstrated that almost all the severity scores used for CAP could be also valuable for HAP [45]. A more recent study focused only on the CURB-65 score, named for the risk factors measured (each scoring 1 point): confusion of new onset, urea $\geqslant 7 \mathrm{mmol} \cdot \mathrm{L}^{-1}$ (blood urea nitrogen $>19 \mathrm{mg} \cdot \mathrm{dL}^{-1}$ ), respiratory rate $\geqslant 30$ breaths per minute, systolic blood pressure $(\mathrm{BP}) \leqslant 90 \mathrm{mmHg}$ or diastolic BP $\leqslant 60 \mathrm{mmHg}$ and age $\geqslant 65$ years [46]. A score $\geqslant 2$ could help to stratify patients with an increased risk of mortality [47]. The use of this severity score in HAP needs further validation, but it could be an important tool to improve the delivery of care for pneumonia patients and to identify early those patients who could deteriorate and need mechanical ventilation [16].

\section{Aetiology and treatment}

The development of HAP and VAP is due to an imbalance between normal host defence and the ability of micro-organisms to colonise and then invade the lower respiratory tract, which is increased in the presence of new points of entrance for the bacteria, such as central venous catheters, nasogastric tubes and tracheostomies. In addition, patients with VAP are usually exposed to more antibiotics than those with HAP, either for past infection or prophylaxis, causing increased colonisation with nosocomial pathogens. Indeed, it has been reported that changes in the oropharyngeal flora in patients with HAP may be delayed, with a persistence of "community-acquired microbes" such as Streptococcus pneumoniae, while patients with VAP have a higher prevalence of nonfermenting and enteric Gram-negative bacilli and methicillin-sensitive S. aureus (MSSA) [13, 48, 49]. However, a recent small retrospective study by FENG et al. [50] of the microbial aetiology of HAP and VAP reported that the leading pathogens were similar in both entities, with a predominance of Acinetobacter baumannii and P. aeruginosa in VAP, and Klebsiella pneumoniae and S. aureus in HAP. Moreover, the authors demonstrated an increase in the MDR bacteria in VAP, with a lower susceptibility of $P$. aeruginosa in VAP compared to HAP, as reported in previous studies [51, 52]. The results of FeNG et al. [50] are in line with those of a less recent prospective observational study by ESPERATTI et al. [13] about ICU-acquired nosocomial pneumonia, where the proportion of relevant pathogens in patients with an isolated organism was similar, suggesting that the cause and the outcome depend on the underlying conditions of the patients rather than previous intubation. To corroborate this hypothesis, this group published another study showing no differences in terms of systemic inflammatory response, severity of nosocomial pneumonia and clinical characteristics between MDR and non-MDR cases. Of note, these findings were similar for VAP and NV-HAP [53]. Therefore, it seems that aetiology and outcomes are similar regardless of whether nosocomial pneumonia is acquired or not during ventilation [13,54], and the appropriate empiric treatment should take into account clinical risk factors for MDR germs, other than the severity of the patient. Consequently, the 2017 European guidelines [2] did not provide a strict distinction in terms of risk factors between VAP and HAP, and it is considered a good practice to apply the recommendations used for VAP in patients with V-HAP and NV-HAP. Treatment should only be based on the risk of mortality and the likelihood of MDR pathogens [2] (table 1). If the risk of MDR is low with a mortality risk $<15 \%$, monotherapy with a narrow-spectrum antibiotic against nonresistant Gram-negative bacteria and MSSA would be an effective choice. However, in patients with a high risk of mortality, a single Gram-negative agent active against Pseudomonas would be an effective choice (except aztreonam, which does not cover Gram-positive bacteria) if the patient is not in septic shock. Conversely, patients with septic shock should always be treated with two antipseudomonal antibiotics, including an antipseudomonal $\beta$-lactam (imipenem, meropenem, cefepime, piperacillin/tazobactam, ceftazidime and aztreonam) plus a second agent such as an aminoglycoside (preferably amikacin, which is the most active agent in many ICUs) or an antipseudomonal quinolone (ciprofloxacin or levofloxacin). Note that in ICUs with high rates of MRSA, linezolid or vancomycin should be added. Similarly, when the prevalence of Acinetobacter spp. and extended-spectrum $\beta$-lactamases (ESBL)-producing Enterobacteriaceae is high, the regimen should include also dual anti-Pseudomonas cover. If Acinetobacter spp. are commonly isolated, the second agent should be colistin. For ESBL-producing germs, a carbapenem may be the best choice, and cefepime and piperacillin/tazobactam only considered based on local susceptibilities. The dual coverage should not be continued after the results of the cultures, except in patients with MDR pathogens. The duration of 
TABLE 1 Empiric treatment for hospital-acquired pneumonia and ventilator-acquired pneumonia according to the 2017 International European Respiratory Society/European Society of Intensive Care Medicine/European Society of Clinical Microbiology and Infectious Diseases/Latin American Thoracic Association guidelines

Low mortality risk $(<15 \%)$

High mortality risk (>15\%)

Non-septic shock

Septic shock

High risk of MDR pathogens
Monotherapy

Narrow-spectrum agent active against nonresistant Gram-negative and MSSA le.g. ertapenem, ceftriaxone, cefotaxime, moxifloxacin or levofloxacin]

Single antipseudomonal agent (e.g. imipenem, meropenem, cefepime, piperacillin/tazobactam, levofloxacin or ceftazidimel

Two antipseudomonal antibiotics: antipseudomonal $\beta$-lactam or cephalosporin

PLUS

aminoglycoside or antipseudomonal quinolone

MRSA: add linezolid or vancomycin

Acinetobacter spp. and ESBL-producing Enterobacteriaceae:

two antipseudomonal antibiotics

Choice of second agent to be based on common isolation:

colistin for Acinetobacter

carbapenem for ESBL-producing germs

MDR: multidrug-resistant; MSSA: methicillin-sensitive Staphylococcus aureus; MRSA: methicillin-resistant Staphylococcus aureus; ESBL: extended-spectrum $\beta$-lactamases.

treatment should be based on the clinical response, the presence of an inappropriate initial empiric therapy or MDR organisms and serial measurement of biomarkers. Usually, 7-8 days are effective in patients without immunodeficiency, cystic fibrosis, empyema, lung abscess, cavitation or necrotising pneumonia and with a good clinical response to therapy.

\section{Future perspectives}

In order to reduce the burden of nosocomial pneumonia [55], the development of new criteria to improve hospital surveillance and identify patients with HAP [56] subtypes are promising tools to monitor and investigate such disease.

New molecular diagnostic techniques will allow a rapid identification of the microbiological aetiology in presence of clinical suspicion of nosocomial pneumonia. Detection of pathogens through more sensitive and specific methods will lead to a better definition of nosocomial pneumonia subtypes, especially NV-HAP. In the light of this view, many tools have been tested in clinical practice [57]. In future, the use of fluorescence in situ hybridisation will identify the pattern of antibiotic susceptibility, thus improving the administration of appropriate drugs as well as limiting their unnecessary use. Furthermore, the electronic nose appears to be a promising technology which will allow the noninvasive identification of specific pathogens using real-time exhaled gas from the ventilator, potentially leading to a rapid and accurate start of antimicrobic therapy in both V-HAP and VAP.

The implementation of a test to discriminate the immune system response (pro-inflammatory versus anti-inflammatory) will allow the identification of patients with different features that will take advance of specific host-targeted therapy [24]. Accordingly, the pathophysiological hypothesis of nosocomial pneumonia based on respiratory dysbiosis (caused by antibiotics, corticosteroids, sedation, supine positioning and acid-suppressing medication) will open the route for further therapies aiming at microbiota restoration, as observed in other diseases $[58,59]$. Ongoing research will shed light on the effectiveness of further therapies, which will improve antimicrobic delivery (e.g. nebulisers [60]) or increase the spectrum of sensitivity of MDR pathogens (e.g. new antimicrobials [61]). Finally, the development and implementation of therapeutic drug monitoring in clinical practice will help clinicians to prescribe the optimal antimicrobic dose, thus allowing the precise administration of drugs to get a specific concentration at a targeted site, in order to improve their effectiveness and reduce any systemic toxicity [60].

\section{Conclusion}

In summary, nosocomial pneumonia is a complex disease, affecting a significant proportion of hospitalised patients and associated with dramatic clinical complications. Although the pathophysiology of nosocomial pneumonia subtypes has not been clearly investigated, no differences have been demonstrated regarding 
the underlying microbiological pathway. However, an epidemiological study showed a significant difference of mortality among the subtypes and identified V-HAP as a worse entity than VAP. Although different flow charts have been proposed to diagnose nosocomial pneumonia subtypes, and many therapies have been suggested in international guidelines, the emergence of MDR pathogens is challenging the care of such patients. New diagnostic and therapeutic tools have been gaining consent among the scientific community and there is hope for the coming years. Considering the worrisome data on mortality of patients with nosocomial pneumonia, the authors of this review strongly believe that prospective studies focused on this subject are urgently needed.

Conflict of interest: M.S. Vallecoccia has nothing to disclose. C. Dominedò has nothing to disclose. S.L. Cutuli has nothing to disclose. I. Martin-Loeches reports personal fees from MSD, outside the submitted work. A. Torres has nothing to disclose. G. De Pascale has nothing to disclose.

\section{References}

$1 \quad$ Kalil AC, Metersky ML, Klompas M, et al. Management of adults with hospital-acquired and ventilator-associated pneumonia: 2016 clinical practice guidelines by the Infectious Diseases Society of America and the American Thoracic Society. Clin Infect Dis 2016; 63: e61-e111.

2 Torres A, Niederman MS, Chastre J, et al. International ERS/ESICM/ESCMID/ALAT guidelines for the management of hospital-acquired pneumonia and ventilator-associated pneumonia: Guidelines for the management of hospital-acquired pneumonia (HAP)/ventilator-associated pneumonia (VAP) of the European Respiratory Society (ERS), European Society of Intensive Care Medicine (ESICM), European Society of Clinical Microbiology and Infectious Diseases (ESCMID) and Asociacion Latinoamericana del Tórax (ALAT). Eur Respir J 2017; 50: 1700582 .

3 Ferrer M, Torres A. Epidemiology of ICU-acquired pneumonia. Curr Opin Crit Care 2018; 24: 325-331.

4 Timsit JF, Esaied W, Neuville M, et al. Update on ventilator-associated pneumonia. F1000Res 2017; 6: 2061.

5 Wang L, Li X, Yang Z, et al. Semi-recumbent position versus supine position for the prevention of ventilator-associated pneumonia in adults requiring mechanical ventilation. Cochrane Database Syst Rev 2016; 1: CD009946.

6 Valencia M, Ferrer M, Farre R, et al. Automatic control of tracheal tube cuff pressure in ventilated patients in semirecumbent position: a randomized trial. Crit Care Med 2007; 35: 1543-1549.

7 Ranzani OT, De Pascale G, Park M. Diagnosis of nonventilated hospital-acquired pneumonia: how much do we know? Curr Opin Crit Care 2018; 24: 339-346.

8 Forel JM, Voillet F, Pulina D, et al. Ventilator-associated pneumonia and ICU mortality in severe ARDS patients ventilated according to a lung-protective strategy. Crit Care 2012; 16: R65.

9 Douglas IS. Pulmonary infections in critical/intensive care - rapid diagnosis and optimizing antimicrobial usage. Curr Opin Pulm Med 2017; 23: 198-203.

10 Dudeck MA, Horan TC, Peterson KD, et al. National Healthcare Safety Network report, data summary for 2011, device-associated module. Am J Infect Control 2013; 41: 286-300.

11 Koulenti D, Tsigou E, Rello J. Nosocomial pneumonia in 27 ICUs in Europe: perspectives from the EU-VAP/CAP study. Eur J Clin Microbiol Infect Dis 2017; 36: 1999-2006.

12 Giuliano KK, Baker D, Quinn B. The epidemiology of nonventilator hospital-acquired pneumonia in the United States. Am J Infect Control 2018; 46: 322-327.

13 Esperatti M, Ferrer M, Theessen A, et al. Nosocomial pneumonia in the intensive care unit acquired by mechanically ventilated versus nonventilated patients. Am J Respir Crit Care Med 2010; 182: 1533-1539.

14 De Pascale G, Ranzani OT, Nseir S, et al. Intensive care unit patients with lower respiratory tract nosocomial infections: the ENIRRIs project. ERJ Open Res 2017; 3: 00092-2017.

15 Micek ST, Chew B, Hampton N, et al. A case-control study assessing the impact of nonventilated hospital-acquired pneumonia on patient outcomes. Chest 2016; 150: 1008-1014.

16 Talbot GH, Das A, Cush S, et al. Evidence-based study design for hospital-acquired bacterial pneumonia and ventilator-associated bacterial pneumonia. J Infect Dis 2019; 219: 1536-1544.

17 Torres A. ICU-acquired pneumonia: is it time to use this term? Curr Opin Crit Care 2018; 24: 323-324.

18 Ranzani OT, Senussi T, Idone F, et al. Invasive and non-invasive diagnostic approaches for microbiological diagnosis of hospital-acquired pneumonia. Crit Care 2019; 23: 51.

19 Muscedere JG, Shorr AF, Jiang X, et al. The adequacy of timely empiric antibiotic therapy for ventilator-associated pneumonia: an important determinant of outcome. J Crit Care 2012; 27: 322.e7-14.

20 Davey PG, Marwick C. Appropriate vs. inappropriate antimicrobial therapy. Clin Microbiol Infect 2008; 14: Suppl. $3,15-21$.

21 Luna CM, Aruj P, Niederman MS, et al. Appropriateness and delay to initiate therapy in ventilator-associated pneumonia. Eur Respir J 2006; 27: 158-164.

22 Pileggi C, Mascaro V, Bianco A, et al. Ventilator bundle and its effects on mortality among ICU patients: a meta-analysis. Crit Care Med 2018; 46: 1167-1174. 10.1097/CCM.0000000000003136.

23 Lyons PG, Kollef MH. Prevention of hospital-acquired pneumonia. Curr Opin Crit Care 2018; 24: 370-378

24 Roquilly A, Torres A, Villadangos JA, et al. Pathophysiological role of respiratory dysbiosis in hospital-acquired pneumonia. Lancet Respir Med 2019; 7: 710-720.

25 Nakagawa NK, Franchini ML, Driusso P, et al. Mucociliary clearance is impaired in acutely ill patients. Chest 2005; 128: 2772-2777.

26 Magiorakos AP, Srinivasan A, Carey RB, et al. Multidrug-resistant, extensively drug-resistant and pandrug-resistant bacteria: an international expert proposal for interim standard definitions for acquired resistance. Clin Microbiol Infect 2012; 18: 268-281. 
27 Bassetti M, Righi E, Vena A, et al. Risk stratification and treatment of ICU-acquired pneumonia caused by multidrug-resistant/extensively drug-resistant/pandrug-resistant bacteria. Curr Opin Crit Care 2018; 24: 385-393.

28 Cillóniz C, Dominedò C, Torres A. An overview of guidelines for the management of hospital-acquired and ventilator-associated pneumonia caused by multidrug-resistant Gram-negative bacteria. Curr Opin Infect Dis 2019; 32: $656-662$.

29 Leroy O, Jaffré S, D’Escrivan T, et al. Hospital-acquired pneumonia: risk factors for antimicrobial-resistant causative pathogens in critically ill patients. Chest 2003; 123: 2034-2042.

30 Bouza E, Giannella M, Bunsow E, et al. Ventilator-associated pneumonia due to meticillin-resistant Staphylococcus aureus: risk factors and outcome in a large general hospital. J Hosp Infect 2012; 80: 150-155.

31 Wooten DA, Winston LG. Risk factors for methicillin-resistant Staphylococcus aureus in patients with community-onset and hospital-onset pneumonia. Respir Med 2013; 107: 1266-1270.

32 Montero M, Sala M, Riu M, et al. Risk factors for multidrug-resistant Pseudomonas aeruginosa acquisition. Impact of antibiotic use in a double case-control study. Eur J Clin Microbiol Infect Dis 2010; 29: 335-339.

33 Ekren PK, Ranzani OT, Ceccato A, et al. Evaluation of the 2016 Infectious Diseases Society of America/American Thoracic Society guideline criteria for risk of multidrug-resistant pathogens in patients with hospital-acquired and ventilator-associated pneumonia in the ICU. Am J Respir Crit Care Med 2018; 197: 826-830.

34 Martin-Loeches I, Deja M, Koulenti D, et al. Potentially resistant microorganisms in intubated patients with hospital-acquired pneumonia: the interaction of ecology, shock and risk factors. Intensive Care Med 2013; 39: 672-681.

35 Blot S, Depuydt P, Vogelaers D, et al. Colonization status and appropriate antibiotic therapy for nosocomial bacteremia caused by antibiotic-resistant Gram-negative bacteria in an intensive care unit. Infect Control Hosp Epidemiol 2005; 26: 575-579.

36 Drakulovic MB, Bauer TT, Torres A, et al. Initial bacterial colonization in patients admitted to a respiratory intensive care unit: bacteriological pattern and risk factors. Respiration 2001; 68: 58-66.

37 Kelly DN, Martin-Loeches I. Comparing current US and European guidelines for nosocomial pneumonia. Curr Opin Pulm Med 2018; 25: 263-270.

38 Kumar A, Safdar N, Kethireddy S, et al. A survival benefit of combination antibiotic therapy for serious infections associated with sepsis and septic shock is contingent only on the risk of death: a meta-analytic/meta-regression study. Crit Care Med 2010; 38: 1651-1664.

39 Korkmaz Ekren P, Basarik Aydogan B, Gurgun A, et al. Can fiberoptic bronchoscopy be applied to critically ill patients treated with noninvasive ventilation for acute respiratory distress syndrome? Prospective observational study. BMC Pulm Med 2016; 16: 89.

40 Lucangelo U, Vassallo FG, Marras E, et al. High-flow nasal interface improves oxygenation in patients undergoing bronchoscopy. Crit Care Res Pract 2012; 2012: 506382.

41 Scala R, Naldi M, Maccari U. Early fiberoptic bronchoscopy during non-invasive ventilation in patients with decompensated chronic obstructive pulmonary disease due to community-acquired-pneumonia. Crit Care 2010; 14: R80.

42 Baumann HJ, Klose H, Simon M, et al. Fiber optic bronchoscopy in patients with acute hypoxemic respiratory failure requiring noninvasive ventilation - a feasibility study. Crit Care 2011; 15: R179.

43 Asti L, Bartsch SM, Umscheid CA, et al. The potential economic value of sputum culture use in patients with community-acquired pneumonia and healthcare-associated pneumonia. Clin Microbiol Infect 2019; 25: 1038 e1-1038.e9.

44 Naidus EL, Lasalvia MT, Marcantonio ER, et al. The diagnostic yield of noninvasive microbiologic sputum sampling in a cohort of patients with clinically diagnosed hospital-acquired pneumonia. J Hosp Med 2018; 13: 34-37.

45 Al-Badawy TH, Abouelela AM, Abdel Kawi MAG. Predictive value of different scoring systems for critically ill patients with hospital acquired pneumonia. Egypt J Chest Dis Tuberc 2016; 65: 757-763.

46 Lim WS, van der Eerden MM, Laing R, et al. Defining community acquired pneumonia severity on presentation to hospital: an international derivation and validation study. Thorax 2003; 58: 377-382.

47 Oktariani N, Pitoyo C, Singh G, et al. CURB 65 score as a predictor of early mortality in hospital-acquired pneumonia. Egypt J Chest Dis Tuberc 2019; 68: 231.

48 Weber DJ, Rutala WA, Sickbert-Bennett EE, et al. Microbiology of ventilator-associated pneumonia compared with that of hospital-acquired pneumonia. Infect Control Hosp Epidemiol 2007; 28: 825-831.

49 Sopena N, Sabrià M. Multicenter study of hospital-acquired pneumonia in non-ICU patients. Chest 2005; 127: 213-219.

50 Feng DY, Zhou YQ, Zou XL, et al. Differences in microbial etiology between hospital-acquired pneumonia and ventilator-associated pneumonia: a single-center retrospective study in Guang Zhou. Infect Drug Resist 2019; 12: 993-1000.

51 Djordjevic ZM, Folic MM, Jankovic SM. Distribution and antibiotic susceptibility of pathogens isolated from adults with hospital-acquired and ventilator-associated pneumonia in intensive care unit. J Infect Public Health 2017; 10: 740-744

52 Zhao T, Liu Y, Cao B, et al. Prospective multicenter study of pathogen distributions in early-onset and late-onset hospital-acquired pneumonia in China. Antimicrob Agents Chemother 2013; 57: 6404-6405.

53 Di Pasquale M, Ferrer M, Esperatti M, et al. Assessment of severity of ICU-acquired pneumonia and association with etiology. Crit Care Med 2014; 42: 303-312.

54 Ferrer M, Difrancesco LF, Liapikou A, et al. Polymicrobial intensive care unit-acquired pneumonia: prevalence, microbiology and outcome. Crit Care 2015; 19: 450.

55 Niederman MS, Martin-Loeches I, Torres A. The research agenda in VAP/HAP: next steps. Intensive Care Med 2017; 43: 1389-1391.

56 Ji W, McKenna C, Ochoa A, et al. Development and assessment of objective surveillance definitions for nonventilator hospital-acquired pneumonia. JAMA Netw Open 2019; 2: e1913674.

57 Douglas IS. New diagnostic methods for pneumonia in the ICU. Curr Opin Infect Dis 2016; 29: 197-204.

58 Moayyedi P, Surette MG, Kim PT, et al. Fecal microbiota transplantation induces remission in patients with active ulcerative colitis in a randomized controlled trial. Gastroenterology 2015; 149: 102-109. 
59 van Nood E, Vrieze A, Nieuwdorp M, et al. Duodenal infusion of donor feces for recurrent Clostridium difficile. $N$ Engl J Med 2013; 368: 407-415.

60 Niederman MS, Chastre J, Corkery K, et al. BAY41-6551 achieves bactericidal tracheal aspirate amikacin concentrations in mechanically ventilated patients with Gram-negative pneumonia. Intensive Care Med 2012; 38: 263-271.

61 Vincent JL, Bassetti M, François B, et al. Advances in antibiotic therapy in the critically ill. Crit Care 2016; 20: 133 . 